\title{
AS COMPETÊNCIAS NECESSÁRIAS PARA O ENGENHEIRO DE PETRÓLEO NO CONTEXTO DIGITAL E GLOBALIZADO
}

DOI: 10.37702/2175-957X.COBENGE.2021.3458

Maria Pontes Pedrosa Peczek - mariapedrosa@id.uff.br

Universidade Federal Fluminense

Rua Professor Lara Vilela 191

24210-590 - Niterói - RJ

Gabriel Maia Sousa - gabrielmaiasousa@id.uff.br

Universidade Federal Fluminense

Rua Antonio Alves Ferreira 40

37170-000 - Boa Esperança - MG

Julia Silva Cavalcante de Aguiar - juliaaguiar@id.uff.br

Universidade Federal Fluminense

Rua Otávio Carneiro 43

24230-190 - Niterói - RJ

Maria Eduarda Melentovytch Ribeiro de Castro - maria_melentovytch@id.uff.br Universidade Federal Fluminense

Rua Jangadeiros 28

22420-010 - Rio de Janeiro - RJ

Geraldo de Souza Ferreira - geraldoferreira@id.uff.br

Universidade Federal Fluminense

Rua Passo da Pátria 156

24210-240 - Niterói - RJ

Resumo: Com a consolidação da indústria 4.0, em um contexto cada vez mais globalizado e com transformações em um ritmo mais acelerado, torna-se importante analisar como se comporta a dinâmica industrial e do mercado de trabalho. No geral, o que se observa é uma busca por profissionais cada vez mais qualificados, tanto com relação às ditas competências digitais quanto às soft skills. Entretanto, nesse mesmo cenário, nota-se que as empresas podem ser mais motivadas para o investimento em suas equipes, em paralelo ao fato de que as universidades podem criar um ambiente mais propício à preparação de alunos 


\section{(C. COBENGE e IV Simpósio Internacional

para lidarem com as demandas contemporâneas. O presente trabalho aborda os desafios inerentes à formação em Engenharia de Petróleo nesse contexto de demanda por novas e diversas competências.

Palavras-chave: Educação. Engenharia. Petróleo. Indústria 4.0. Competências. 


\section{AS COMPETÊNCIAS NECESSÁRIAS PARA O ENGENHEIRO DE PETRÓLEO NO CONTEXTO DIGITAL E GLOBALIZADO}

\section{INTRODUÇÃO}

Após três processos transformadores, as chamadas Revoluções Industriais, que, respectivamente, mecanizaram, expandiram e automatizaram a produção, emergiu, então, a necessidade de adaptar essa automatização para o processo de tomada de decisões, aspecto que, somado a uma busca por maior produtividade e eficiência, levou a um novo processo de reestruturação e, portanto, à Quarta Revolução Industrial (LUND, 2019). Essa nova abordagem se centraliza na digitalização para reinventar produtos e serviços, impactando a forma como vivemos, relacionamo-nos e trabalhamos, tendo, por sua vez, alcance e complexidade consideráveis (COLCHER, 2019).

Dessa forma, há, hoje, significativo aumento no fluxo digital, o que torna a digitalização um driver do aprofundamento da globalização: diversos recursos online remodelaram a forma como nos comunicamos, colaboramos e aprendemos. Assim, o cenário atual é marcado por avanços sem precedentes no campo das tecnologias da informação e comunicação, impulsionados por progressos em áreas como computação, eletrônica e sistemas digitais integrados (FRANK \& MOORE, 2010). Surge, portanto, a necessidade de rápida adaptação das organizações, com suas oportunidades, desafios e instabilidades, que, mesmo permitindo às empresas adotarem modelos de negócios menos intensivos em capital e otimizar seus processos e lucros, ainda apresenta novos milestones para o futuro do mundo do trabalho.

A indústria do futuro, então, começa a ser pautada no uso intensivo de tecnologias associadas à digitalização, ciência de dados, big data, machinelearning, inteligência artificial, sensoriamento, robótica e manufatura aditiva. Sobretudo no Brasil, essas tecnologias representam oportunidades para o país se tornar mais competitivo, melhorar sua produtividade e adaptar a força de trabalho às necessidades atuais, aumentando assim sua eficiência.

O presente trabalho tem, portanto, como objetivo abordar as competências que os engenheiros de petróleo necessitam consolidar para lidar com as transformações distintas que afetam esta nova realidade que permeia o mundo produtivo e as organizações empresariais.

\section{A IMPLANTAÇÃO DA GLOBALIZAÇÃO}

O termo globalização, ainda que consideravelmente comum no cotidiano atual, começou a ser difundido na década de 80 , por meio da imprensa financeira internacional (RIBEIRO, 2002). De fato, durante as décadas de 80 e 90, a globalização industrial transformou tanto a manufatura quanto 0 varejo e, desde então, nota-se um aprofundamento das transformações relacionadas ao dinamismo do capitalismo internacional. A globalização passou, dessa forma, a se relacionar à difusão e à mundialização de mercados, bem como sua crescente integração. Sendo assim, o avanço de tal processo transcende a sua origem, de fenômenos meramente econômicos, e passa a abranger também as esferas política, social e cultural (DELUIZ, 2004). 
Com sua expansão, há um acirramento na competição intercapitalista, de modo que as empresas inseridas no contexto da globalização, passam a buscar estratégias para se manterem competitivas (BAUMAN, 1999). Dessa forma, na era moderna, o processo de globalização se expande, efetiva e se modifica, maximizado pela digitalização. Portanto, ainda que a percepção popular seja de que o processo de globalização chegou a um ponto de saturação e consequente estagnação, o que se nota é uma sociedade ainda sujeita a mudanças, as quais são explicitadas no aumento do fluxo digital. Tal fator, que se mostrava quase imperceptível no passado, hoje, exerce um impacto mais amplo no crescimento econômico, possibilitando às empresas chegar aos mercados globais com modelos de negócios menos intensivos em capital, mesmo que com novos riscos e desafios políticos.

Dentre as indústrias energéticas, a de óleo e gás é a única que pode ser classificada como uma indústria energética global (MATHIAS, 2008). Ademais, um exemplo de como tal perfil ganha força é o de dezessete empresas, nacionais e internacionais, de petróleo trabalhando juntas por meio do Fórum Econômico Mundial para chegar a um acordo sobre especificações de aquisição padronizadas pilotos para válvulas esfera, árvores de natal molhadas e aparelhagem de baixa tensão (HANDSCOMB et al, 2016).

\section{UM NOVO CENÁRIO PARA AS EMPRESAS E PARA O MUNDO DO TRABALHO}

É notório que as organizações já fizeram um grande avanço no desenvolvimento de estratégias, com uma nova onda de disrupções pautadas por inteligência artificial, dados e análises. Essas disrupções, por sua vez, demandam novas abordagens para gerenciar, capacitar e alinhar a força de trabalho à nova realidade.

Além do considerado novo perfil global das indústrias e com a transformação digital já significativamente consolidada, percebe-se que é o momento para as empresas repensarem suas estruturas. Assim, com os rápidos avanços tecnológicos acerca de dados e análise, a nova questão é como integrar tais recursos às operações e estratégias e, ainda, como se posicionar em um cenário em que os recursos de análise remodelam rapidamente a concorrência.

Nesse contexto, é importante reconhecer que os dados não estão apenas mais volumosos, mas também mais ricos e diversificados, de modo que se tornaram a nova classe de ativos corporativos - e a melhor forma para as empresas tanto gerarem quanto acessarem os mesmos é por meio da digitalização (LUND, 2019). Isso se dá por consequência de uma nova era em que os mundos físico e digital estão cada vez mais conectados.

Ademais, o capital humano provou ser uma das maiores barreiras à utilização de todo o potencial dos dados e análises. Então, em resposta à expressiva disrupção digital, começa-se a repensar como valorizar e investir em sua força de trabalho, de modo que o cenário corporativo passa a buscar o desenvolvimento de uma força de trabalho mais flexível, adaptável e valiosa (SCHRAGE, 2020).

Dessa forma, a discussão acerca de adaptar-se ao digital, que impunha-se como a diferença entre liderar a nova onda de inovação da indústria ou ficar para trás, pode ser considerada ultrapassada: a atenção agora volta-se a como otimizar a interação cyber física, sobretudo no que diz respeito aos profissionais contemporâneos e os que virão. 


\section{AS MUDANÇAS GERACIONAIS E A DIGITALIZAÇÃO - NOVAS PERSPECTIVAS}

Em 2016, a consultora McKinsey já apontava que os chamados Millennials - as pessoas nascidas após o início da década de 80 e aproximadamente o fim do século XX constituiriam majoritariamente a força de trabalho nos Estados Unidos no início da década de 2020 e que, à época já haviam começado sua jornada às posições gerenciais e até mesmo executivas (HANDSCOMB et al 2016). Como nativos digitais, essa geração teve a internet como ferramenta disponível desde os seus anos de formação, o que fez das suas interações com a mesma algo natural. Tal circunstância abriu margem para 0 desenvolvimento de uma mentalidade coletiva, interconectada e mais expandida, que celebra a colaboração e atribui igual valor para as interações virtuais e físicas.

Essa mudança demográfica reflete exigências de mudanças no ambiente de trabalho por parte dos funcionários, além de expressarem preocupações sobre o papel das empresas de óleo e gás na sociedade. Não só isso, ao passo que os millennials passam a ocupar as empresas, trazem consigo suas próprias expectativas acerca de tecnologia, colaboração, ritmo e responsabilidade - o que faz com que os membros de tal geração comecem a mudar as normas sociais e operacionais das corporações.

Sendo assim, as empresas líderes projetarão um ambiente que atenda às expectativas dos líderes millennials e que tem por base três características principais: (i) estruturas de emprego mais flexíveis, (ii) um novo ambiente de trabalho e cultura - como, por exemplo, a aplicação de ferramentas de mídia social no ambiente corporativo, ao invés de ferramentas tradicionais de intranet e compartilhamento de arquivos - e (iii) uma pegada externa positiva (HANDSCOMB et al, 2016).

Por exemplo, ao passo que empresas de óleo e gás adotem formas de trabalho mais ágeis, elas podem atrair mais talentos dentre os millennials, os quais, por sua vez, vão acelerar ainda mais a adoção de tecnologias digitais - o que pode facilitar 0 desmembramento de grandes centros corporativos, que, por sua vez, possibilita ainda mais a agilização do trabalho. Isso é importante uma vez que, numa economia virtualizada, é mais vantajoso se organizar próximo aos mercados do que às supplychains (FRANK \& MOORE, 2010). Portanto, é possível que as empresas de óleo e gás necessitem mudanças mais profundas na busca por atender às demandas por trabalho significativo e responsabilidade social, de modo a atrair a nova geração de engenheiros e líderes.

\section{$5 \quad$ O QUE SE BUSCA ATUALMENTE DO PROFISSIONAL DE ENGENHARIA?}

Ao passo que a indústria endossa seu perfil digitalizado e global, ela transforma o mercado de trabalho, de forma que os profissionais devem se adaptar às novas exigências. Surge, assim, a busca pelo domínio de um conjunto de conhecimentos, competências e tecnologias que, em sinergia, proporcionam diversos benefícios ao setor.

Desse modo, a formação acadêmica torna-se válida, neste novo contexto, desde que associada à aquisição de aprendizados mais concretos em ambiente fora da sala de aula, de modo a se efetivarem diferenciais nos currículos (GILCHRIST, 2016). Isso porque é possível que haja gradativa saturação do mercado com pessoas que possuem graduação, mas com baixas qualificações e poucas diferenciações (BBC NEWS, 2016). Sendo assim, o mercado de trabalho apresenta uma base técnica uniformizada e com mais profissionais que a possuem, aumentando a competitividade e tornando mais rígidos os critérios de seleção. 
Ainda, é válido reconhecer que, as tecnologias de dados e análises, oriundas do avanço da Transformação Digital, beneficiam o campo da inovação de processos, uma vez que auxiliam as organizações a estruturar suas equipes, recursos e fluxos de trabalho. Por consequência, torna-se importante a discussão acerca de questões como a complementaridade de competências (HENKE, 2016).

De acordo com Howeset al. (2017), habilidades, por definição, são a capacidade de fazer algo bem, envolvendo ideias, coisas e/ou pessoas, em uma área ou domínio que é adquirido ou desenvolvido por meio de treinamento ou experiência. Assim, ainda que apenas recentemente tenham sido identificadas como essenciais por empresas de óleo e gás, em particular, as habilidades para garantir o uso apropriado da ciência de dados para interpretar resultados como da inteligência artificial são cruciais (CLEMENS \& GRUBER, 2020). O setor petrolífero, portanto, passa a buscar profissionais que consigam utilizar ferramentas informacionais avançadas para aplicá-las à melhoria de processos tanto no downstream quanto no upstream - por exemplo, no mapeamento de dados geofísicos a fim de se ter mais exatidão nas perfurações.

Dessa forma, profissionais da engenharia de petróleo que tenham bom domínio das ditas competências digitais são os que obterão destaque no mercado de trabalho bem como controlarão o futuro do setor. Tal aspecto reforça a atual "jornada de bytes à barris" (DELOITTE, 2017).

Por outro lado, Schenewer \& Alman (1995) salientam que, além do uso do computador, isto é, as competências digitais, o trabalho em equipe, o pensamento crítico e aspectos interdisciplinares são pontos relevantes a serem inseridos no currículo de Engenharia de Petróleo, de modo que o estudante e futuro profissional disponha não só do conhecimento técnico, mas também das habilidades interpessoais e digitais para se destacar no mercado.

Nesse contexto, as chamadas soft skills tornam-se cada vez mais importantes. Essas são a coleção de habilidades de gerenciamento de pessoas, como competências de comunicação ou habilidades que conectam o indivíduo com os aspectos técnicos do trabalho e que permitem entregar o melhor resultado possível. Ademais, é válido notar que essa gama de competências é fluida e pode mudar de acordo com a situação profissional e da cultura da empresa em questão. Mesmo assim, é possível dividir as soft skills em cinco macro grupos: comunicação, colaboração, persuasão, tomada de decisão e auto gerenciamento (DALRYMPLE \& DALRYMPLE, 2017).

Portanto, torna-se importante a constante busca pela adaptação às exigências de um mercado de trabalho cada vez mais dinâmico e pautado no diferencial competitivo, isto é, nas competências adquiridas além do âmbito técnico adquirido na graduação. Particular destaque pode ser dado à Schneider Electric, que mantém um sistema de referência continuamente atualizado no qual são catalogadas as habilidades necessárias para cada organização ao redor do mundo. Além disso, a empresa busca a opinião dos funcionários sobre quais habilidades eles têm mais interesse em adquirir (SCHRAGE, 2020).

\section{PERSPECTIVAS DE INTEGRAÇÃO DA NOVA GERAÇÃO AO MERCADO DE TRABALHO NO CONTEXTO DA DIGITALIZAÇÃO DA CADEIA PRODUTIVA DO PETRÓLEO}

Atualmente, mais do que nunca, mostra-se indispensável dar continuidade a estudos e aprimoramentos, para além das instituições superiores.Isso ocorre pois as funções exercidas por um engenheiro de petróleo requerem que ele esteja constantemente aprimorando as suas competências para que se destaque no mercado de trabalho. Os 
Millennials, que já começam a compor a maior parte da força de trabalho, já perceberam isso: uma pesquisa realizada pela Manpower em 2016, nos Estados Unidos, mostrou que $93 \%$ da nova geração da força de trabalho está disposta a bancar novos treinamentos e capacitações para aprimorar suas habilidades (SELINGO, 2017).

Entretanto, um longo caminho ainda deve ser percorrido para que as novas demandas por competências sejam supridas. No que tange ao âmbito de aprendizagem, o modelo de ensino empregado pelas universidades não mudou muito ao longo das décadas. Nota-se, então, um distanciamento entre o modelo acadêmico atual - e convencional - e as exigências e expectativas do mercado (PECZEK et al., 2019). Além disso, a educação superior tem múltiplas funções na sociedade, inclusive a de expandir as perspectivas dos estudantes e prepará-los para o mundo.

Sendo assim, os empregadores questionam cada vez mais se o diploma puramente continua sendo um sinal de prontidão para o trabalho em um cenário em que multiplicamse os graduados, mas sua aquisição de soft skills, bem como as digitais não acompanha a demanda (SELINGO, 2017). Ainda, a sociedade e seus desdobramentos se mostram cada vez mais complexos, tornando os planos de carreira possivelmente caóticos - soma-se a isso, as mudanças de paradigma inerentes a uma maior presença de Millennials -, de modo que as competências consideradas necessárias possuem um perfil fluido e mudam com 0 tempo.

Portanto, surge a necessidade de adaptar o ensino superior para que se consiga capacitar da melhor forma os futuros profissionais. Para isso, o curso deve assimilar a dualidade do aprendizado teórico e prático além de levar para dentro da sala de aula conhecimentos e ensinamentos do cotidiano de trabalho. Desse modo, propicia-se uma experiência integrada, na qual há um enriquecimento não só das competências técnicas, mas também da perspectiva acerca do dinamismo das soft skills e de quais habilidades digitais são mais utilizadas. A inserção de disciplinas e propostas extracurriculares que abordem essas duas categorias de competências também se mostra válida - por exemplo, o curso de Engenharia de Petróleo da Universidade Federal Fluminense passou, em 2019, a oferecer uma disciplina optativa sobre Inteligência Artificial, demonstrando essa necessidade das universidades se adaptarem.

As empresas, por sua vez, não investem de fato no desenvolvimento dos seus funcionários, que acabam por serem responsáveis pela sua própria carreira e aprimoramento (SELINGO, 2017). Portanto, tal carência de investimento empresarial na força de trabalho, somada a um mercado cada vez mais competitivo e exigente, também reflete o início dessa transição na relação do mercado de trabalho com a universidade e reforça a necessidade de adaptação do ensino superior.

\section{CONSIDERAÇÕES FINAIS}

O ritmo das mudanças está cada vez mais rápido. Não só o volume de dados continua a dobrar a cada três anos (HENKE, 2016), o conhecimento humano dobra a cada treze meses em média (SELINGO, 2017). Além disso, a IBM prevê que nos próximos anos as informações dobrem a cada onze horas devido à expansão da Internet of Things (SELINGO, 2017). Nesse contexto, é notório que os profundos avanços tecnológicos da Indústria 4.0 representam uma significativa disrupção nos modelos industriais que passam a adotar a automação em grande escala e uma maior interação cyber física.

Por sua vez, a digitalização correlaciona-se com tecnologias de dados e recursos analíticos, visto que é um facilitador importante na geração e na coleta de dados. Dessa forma, além das mudanças nos processos e na infraestrutura, a Transformação Digital 
também requer competências e mentalidades relacionadas à ciência de dados e outras tecnologias. Além disso, as soft skills também ganham cada vez mais destaque, de modo que mais de $94 \%$ em 7.000 engenheiros sentem que esse tipo de habilidades foram os fatores mais necessários a serem considerados em uma carreira de engenharia de sucesso (DALRYMPLE \& DALRYMPLE, 2017).

Entretanto, ao combinar as competências pessoais com as ferramentas digitais, ambas necessárias para um bom desempenho do profissional, fica claro que a ampliação das capacidades demandadas pela indústria não está em sincronia com a velocidade do ensino nas universidades, o que reforça a necessidade das universidades de se adaptarem. Portanto, na área de recursos humanos, modernizar a educação e desenvolver competências em consonância com as atuais necessidades do setor petrolífero é fundamental para fortalecer a indústria e melhorar a capacidade de enfrentar a concorrência crescente do mercado de trabalho. Para caminhar nessa direção, mostra-se importante adotar mudanças no ensino: aprimorando os currículos e melhorando as metodologias de ensino com a inserção de ferramentas digitais, por exemplo.

Algumas instituições já começaram a dar os primeiros passos, seja com a inserção de disciplinas ou mesmo com programas como o MicroMasters, nos Estados Unidos, que aceleram a obtenção do mestrado. Projetos como esses ganharam gradativamente mais importância no cenário acadêmico, pois possibilitam que o estudante ou o profissional se desenvolvam de forma mais ágil. Em relação à habilidades pessoais, uma das principais iniciativas existentes hoje é a Soft Skills Council da Society of Petroleum Engineers (SPE), que tem como objetivo reagir à crescente importância da indispensabilidade de auxiliar os profissionais de óleo e gás a desenvolverem suas competências interpessoais e agregar valor às empresas.

No geral, o que se observa é um mercado de trabalho que precisa de profissionais cada vez mais qualificados, tanto acerca das ditas competências digitais quanto das soft skills. Mas, nesse mesmo cenário, muitas empresas ainda não construíram estratégias para investimento em suas equipes, enquanto muitas universidades ainda não estão com os ambientes de ensino-aprendizagem adequados às demandas destes novos tempos.

\section{REFERÊNCIAS}

BAUMAN, Z. Globalização: as consequências humanas. São Paulo: Zahar, 1999.

BBC NEWS BRASIL. Diploma inútil? Por que tantos brasileiros não conseguem trabalho em suas áreas. BBC News Brasil, São Paulo, 04 de novembro de 2016. Disponível em: <https://www.bbc.com/portuguese/brasil-37867638>. Acesso em 11/03/2021.

CLEMENS, T.; GRUBER, M. V. Impact of Digitalization on the Way of Working and Skills Development in Hydrocarbon Production Forecasting and Project Decision Analysis. SPE Res Eval\&Eng 23 (2020): 1358 - 1372. Disponível em: $<$ https://onepetro.org/REE/article-abstract/23/04/1358/451116/Impact-of-Digitalization-onthe-Way-of-Working-and?redirectedFrom=fulltext>. Acesso em: 24, abril 2021.

COLCHER, R. A globalização e a transformação digital: Promessas e desafios de um novo mundo em construção. São Paulo: Scortecci, 2019. 
DALRYMPLE, L. L.; DALRYMPLE, E. D. Empowering Technical Professionals with Effective Soft Skills Leads to A More Successful Business. SPE Annual Technical Conference and Exhibition, San Antonio, Texas, EUA. Outubro, 2017. Disponível em: $<$ https://onepetro.org/SPEATCE/proceedings-abstract/17ATCE/2-

17ATCE/D021S020R006/193160> .Acesso em: 09, março 2021.

DELOITTE. Rewriting the rules for the digital age. Deloitte. [Online]. Deloitte, 2017. Disponível em: <https://www2.deloitte.com/content/dam/Deloitte/global/Documents/ AboutDeloitte/central-europe/ce-global-human-capital-trends.pdf>. Acesso em: 12/03/2021.

DELUIZ, N. A globalização econômica e os desafios à formação profissional. Boletim Técnico do Senac, 2004: 30(3), 73-79. Disponível em: $<$ https://www.bts.senac.br/bts/article/view/500>.

FRANK, M.; MOORE G. The future of work. A new approach to productivity and competitive advantage. Cognizant, 2010.

GILCHRIST, A. (2016). Introducing industry 4.0. In: Industry 4.0 (pp. 195-215). Apress, Berkeley, CA. Disponível em: <https://link.springer.com/chapter/10.1007/978-1-4842-20474_13\#citeas> .Acesso em: 24, abril 2021.

HANDSCOMB, C.; SHARABURA,S.; WOXHOLTH, J. The oil and gas organization of the future. McKinsey \&Company, Setembro, 2016. Seção Oil\&Gas. Setembro de 2016.

HENKE, N;.BUGHIN, J.; CHUI, M.; MANYIKA, J.; SALEH, T.; WISEMAN, B.; SETHUPATHY, G. The age of analytics: Competing in a data-driven world. Mckinsey\&Company. 7, Dezembro, 2016. Disponível em: <https://www.mckinsey.com/business-functions/mckinsey-analytics/our-insights/the-ageof-analytics-competing-in-a-data-driven-world> . Acesso em: 29, março, 2021.

HOWES, C. S.; DALRYMPLE, L.; NELSON, J.; SOLOMON, G.. Delivering Better Business Outcomes with Soft Skills. SPE Annual Technical Conference and Exhibition, San Antonio, Texas, Estados Unidos. Outubro, 2017. Disponível em: <https://onepetro.org/SPEATCE/proceedings-abstract/17ATCE/2-

17ATCE/D021S020R002/193390> .Acesso em: 09, março 2021.

LUND, S. et al. Globalization in transition: The future of trade and value chain. McKinsey \& Company, 16, Janeiro, 2019. McKinsey Global Institute. Disponível em: $<$ https://www.mckinsey.com/featured-insights/innovation-and-growth/globalization-intransition-the-future-of-trade-and-value-chains\#>. Acesso em: 2, março, 2021.

MATHIAS, M. C. P. P. A formação da indústria global de gás natural: definição, condicionantes e desafios. 2008. Dissertação (Tese de Doutorado) Planejamento Energético - UFRJ, RJ. 
PECZEK, M. P. P.; CANTUÁRIA, M. A. G.; FERREIRA, G. S. A Transformação digital e seu impacto na indústria de óleo e gás e na formação em Engenharia de Petróleo: um panorama. COBENGE, Fortaleza, 2019.

RIBEIRO, W. C. Globalização e geografia em Milton Santos. Script Nova, Barcelona, v. 6. n. 124, p. [9], 2002. Disponível em: < http://www.ub.edu/geocrit/sn/sn-124a.htm >.

SCHENEWERK, P.; ALMAN, R. Petroleum engineering education: Innovation to meet the needs of industry. ONEPETRO, 1995. Disponível em: <https://onepetro.org/SPEATCE/ proceedings-abstract/95SPE/All-95SPE/SPE-30655MS/57840>. Acesso em: 12/03/2021.

SCHRAGE, M.; SCHWARTZ, J.; KIRON, D.; JONES, R.; BUCKLEY, N. Opportunity Marketplaces, MIT Sloan Management Review and Deloitte. Abril, 2020. Disponível em: $<$ https://sloanreview.mit.edu/projects/opportunity-marketplaces/> .Acesso em: 22, abril 2021.

SELINGO, J. The future of the degree: how colleges can survive the credential economy. Washington, D.C.: The Chronicle of Higher Education, 2017. Disponível em: <https://store.chronicle.com/products/the-future-of-the-degree>. Acesso em: 09, março 2021.

\title{
THE SKILLS NEEDED BY THE PETROLEUM ENGINEER IN A DIGITAL AND GLOBALIZED CONTEXT
}

\begin{abstract}
With the consolidation of industry 4.0 in an increasingly globalized context and with transformations at a faster pace, it is important to analyze how industrial and job market dynamics behave. In general, what is observed is a search for ever more qualified professionals, both about the so-called digital skills and soft skills. However, in this same scenario, it's noticeable that companies could be more motivated towards investment in their teams, alongside the fact that universities could create a more proper environment to prepare students to deal with contemporary demands. The present work addresses challenges inherent to the formation in Petroleum Engineering in this context of demand for new and diverse skills.
\end{abstract}

Keywords: Education. Engineering. Petroleum. Industry 4.0. Skills. 